\title{
Editorial
}

\section{Pat Croskerry}

\section{The need for cognition and the curse of cognition}

https://doi.org/10.1515/dx-2018-0072

Two studies in this issue focus on aspects of cognition in medical decision making. Chu et al. [1] examine the commonality of cognitive error in cases selected for presentation at morbidity and mortality rounds over a 5-year period, while Schnapp et al. [2] look at the overall incidence of cognitive errors in an emergency department over an 8-month period. Both studies support earlier work in an internal medicine setting that showed cognitive error is common in diagnosis [3], and reflect further effort towards elaborating the cognitive processes underlying clinical decision making. Both are excellent additions to the burgeoning literature on this topic.

Over a decade ago, Groopman's book How Doctors Think [4] was a watershed moment in the willingness of medical people to talk about their thinking, and helped brighten the light that was beginning to shine on diagnostic failure. Yet, a decade later many remain unconvinced. Cognition is an area where medical people tread gently and go carefully, if at all. Some generally steer clear of it under the pretext that how we think does not have a lot to do with medical practice, which should concentrate on knowing the facts. While the emphasis on the acquisition of a comprehensive medical knowledge is beyond debate, knowledge alone is not sufficient for clinical reasoning. Besides knowing what to think we also need to know how to think, which includes understanding the processes that underlie well-calibrated thinking such as how we feel, reason, problem solve and decide. This area has mostly been the remit of cognitive scientists, and while their findings need to be adopted into studies of clinical decision making, knowledge transfer in this area has been slow and continues to meet with resistance.

Lest this be construed as a problem peculiar to cognitive science, we should remind ourselves that the acceptance of a need for evidence-based medicine did not occur until the end of the 20th century. Could we imagine evidence-based physics or evidence-based chemistry being similarly delayed? The belated recognition of the critical role that cognitive processes play in clinical reasoning may be a victim of this same recalcitrance. A psychologist at the last "Diagnostic Error in Medicine (DEM)" meeting, sponsored by the Society to Improve Diagnosis in
Medicine (SIDM) meeting expressed surprise to this writer over a perceived unwillingness to accept the 'slam-dunk' findings in the cognitive sciences literature on the impact of bias on reasoning. Why is something so obvious having difficulty reaching the minds of those who need it? The impact of cognitive biases has been widely acknowledged in all areas of human reasoning, from the humble processes in Bryology [5] to events on the world stage [6, 7]. So, certainly, they apply to diagnosis, yet some explicitly maintain they do not, while others implicitly ignore them.

As an example, at last year's DEM meeting, a discussion was presented of a case of mis-diagnosis. The analysis was detailed, knowledgeable and thoughtful, yet at no point was bias mentioned, even though several possibilities were evident. The goal of the diagnostician and the discussion panel was clearly to get the correct diagnosis (something we have traditionally done in medicine) to the virtual exclusion of any discussion of the cognitive processes through which it had originally failed (something we should now be doing to avoid repetition of mistakes). This was a missed opportunity and we can imagine the contrast between how the case was discussed at a Morbidity and Mortality (M\&M) rounds which endorsed the discussion of cognitive error versus one that did not. The learning experience of the respective residents and faculty would have been very different. We need senior clinicians in this field to understand and accept the critical role that cognitive error plays in diagnostic failure, especially the role of cognitive biases, otherwise we will continue to underestimate it. M\&M rounds are golden opportunities to discuss cognitive error, as Chu et al. [1] note, and, in some departments, this has been made a mandatory part of the discussion of any case presented. However, in the majority of M\&M rounds, discussion of cognitive errors as a primary objective remains a low priority. It seems important to understand why this is so. If these rounds are about trying to understand events in hindsight, and if this includes what people were thinking (as it should), then cognitive errors need discussion; preferably acknowledging hindsight bias and avoiding blame. Many of the common cognitive biases are readily identifiable with practice. Given that cognitive processes are mostly invisible, some speculation about exactly which ones were involved will be necessary, although it is often possible 
to identify what someone was thinking on the strength of their actions as cognition typically precedes behaviour and there is usually a causal relationship.

Common to both studies here $[1,2]$, are methodologies that focus on broad categories of classification that were developed originally by Graber et al. [3]. At the outset, the process of developing a taxonomy is useful for organization and bringing order to things. But, while the family, order, phylum and genus are important for understanding the origins of an animal, it is the species itself that ultimately attracts our interest. More granularity is needed at the clinical interface with the patient so that instead of simply acknowledging problems associated with faulty synthesis or faulty data gathering, we can identify and focus on the actual cognitive biases which are often the root cause of the problems. Describing problems at a broader level tends to focus on proximal issues whereas the problem often originates with distal ones [8]. For example, one might correctly observe that a clinician conducted an 'ineffective, incomplete or faulty history and physical examination' resulting in a 'failure to collect appropriate information from the initial interview and examination'. However, these are proximal errors that may have been caused by attaching too strongly to a presenting symptom at the outset of the examination or interface with the patient, which itself is a distal error perhaps due to a cognitive bias such as anchoring. In fact, any of the basic biases might be the cause of distal error in several of these broad categories. Importantly, solutions for proximal error may not work for distal error. Ultimately, we will learn more from the distal causes of diagnostic failure than the proximal ones.

Perhaps the use of broader categories of error classification reflect an unwillingness to use the word 'bias'. In common use it has a negative connotation. Yet in cognitive science it is simply a neutral descriptor for a piece of behavior, and often a useful shorthand. Does it make any difference if these well-accepted terms are not used? The answer, surely, is yes. Not using them is a denial of their existence, which is irrational. Awareness and recognition of cognitive biases is seen as a hallmark of rationality [9] and failure to do so constitutes irrational behavior, leading to continued failure in dealing with them. Elsewhere, a variety of reasons have been suggested to explain why the findings from cognitive science have such low penetrance in medicine [10].

Perhaps, too, the spectre of cognitive error is daunting to some because even if we can successfully come to terms with it we may not be able to do anything about it. Both studies here [1, 2] cite reports that promulgate the gloom associated with attempts at cognitive bias mitigation
(CBM) usually referred to as 'de-biasing'. However, it is important to get the correct perspective on this as the recognition of bias and its mitigation is probably the most important challenge ahead in dealing with diagnostic failure. Other recent studies reveal a more positive outlook. Graber et al. [11] reviewed a number of studies describing interventions to improve clinical reasoning and decision-making, which included reflective practice and active metacognitive review. Several showed positive, beneficial effects. Importantly, they found 42 studies which included interventions to reduce cognitive error, and another 100 which made suggestions about how it might be reduced. Another recent review found over 40 categories of interventions [12]. Such activity and effort strongly suggest that the importance of the challenge has now gained widespread recognition. Further, a recent systematic review of 28 studies in CBM and dual process thinking led the authors to conclude that it was now time to recommend such cognitive interventions to educators [13]. Yet another systematic review found that the majority of 68 health-related debiasing studies were partially successful [14]. It appears we can now be more optimistic about melioration of cognitive error, and need to look hard at other general strategies to promote good thinking. There is a plan here: step 1 was to raise awareness and understanding of cognitive bias in clinical decision making, step 2 was to examine a variety of candidate strategies for bias mitigation, and step 3 is to develop effective strategies that we can integrate into clinical practice to reduce diagnostic failure.

Diagnosis is about problem solving and problem solving is about thinking. Given the current failure rate of diagnosis of $10-15 \%$, and given the preponderance of cognitive failure, enhancing thinking skills would appear to be a rational strategy. In a meta-analytic study that looked at the effects of interventions that enhanced thinking skills, a jaw-dropping effect size of 0.65 was found - equivalent to moving a class from the 50th to the 74th percentile. The outcome was measured problem solving across the board by empirical tests such as Raven's matrices [15]. The authors of this study and members of the Foundation for Critical Thinking will, no doubt, be surprised to hear that the improvement of problem solving through critical thinking is a myth [16]. It might come down to the definition of critical thinking. It has been defined by the Foundation for Critical Thinking as 'disciplined thinking that is governed by clear intellectual standards: clarity, accuracy, precision, logic, relevance, consistency, logical correctness, completeness and fairness'. It is irrational to argue that these standards are neither vital to thinking skills nor supportive of good thinking styles. 
Improvements in two thinking styles in particular will probably foster better clinical decision making. The first is improved rationality. Not only can rationality be improved by training but we could start off at a higher point by selecting candidates for medical training who have a disposition towards actively open-minded thinking (AOT) [7]. Individual AOT scores are highly correlated with rationality, which may now be measured as the individual's rationality quotient (RQ) [9], which, because it includes intelligence, may be more useful than IQ alone. The second is need for cognition (NFC) [17], a psychological test that measures cognitive motivation. It assesses the extent to which a person looks for and likes engaging in mentally stimulating and cognitively effortful activities. Those who score well on the test are less likely to get bored with mentally challenging tasks, do better on such tasks, reflect more on relevant information, invest more cognitive resources in information processing, and more likely to use critical processing strategies and to actively search for information [18]. In short, they are the opposite of those who are cognitive misers - those who superficially assess problems, and impulsively accept WYSIATI (what you see is all there is) which, as Kahneman notes, can get us into trouble [19]. There are a number of other aspects of individual thinking that impact the calibration of clinical decision making $[12,20]$. Further, with the increasing onus on collaborative care, there is an emerging imperative to develop a raised level of awareness and understanding of decision making in groups, which has the potential to be greater than the sum of its parts.

To the variety of reasons proposed for the low penetrance of cognitive science into clinical decision making [10], might be added the curse of knowledge, i.e. those who understand the processes have failed to reach those who need to apply them. How effectively do proponents of cognitive approaches articulate the findings of cognitive science to students of medicine? The initial introduction of bias to the medical literature followed a standard format of literal descriptions, usually in the dry language of cognitive scientists. More recently, several new strategies have been introduced to make the new lexicon and concepts more palatable. Visual graphics have been used in the presentations of biases (http://www.visualcapitalist.com/ wp-content/uploads/2017/09/cognitive-bias-infographic. html, https://lifehacker.com/this-graphic-explains-20cognitive-biases-that-affect-y-1730901381), and SIDM has engaged the novel technique of cartoon graphics to get the message across (https://www.youtube.com/watch?v=nQr fFNmVfZY\&feature=youtu.be). This idea probably originated with the use of comics. Their graphic images are excellent for conveying understanding of biases and other cognitive failures [21]. Finally, another creative strategy that has recently emerged is through playing "serious games", web-based exercises developed to address cognitive bias in national security analyses [22]. These techniques engage mental imagery to enhance perceptual learning about cognitive biases and other cognitive failures, as well as their mitigation. Perhaps the curse of cognition can be overcome with such tools?

Author contributions: The author has accepted responsibility for the entire content of this submitted manuscript and approved submission.

Research funding: None declared. Employment or leadership: None declared. Honorarium: None declared.

\section{References}

1. Chu D, Xiao J, Shah P, Todd B. How common are cognitive errors in cases presented at emergency medicine resident morbidity and mortality conferences? Diagnosis 2018;5: 143-50.

2. Schnapp BH, Sun JE, Kim JL, Strayer RJ, Shah KH. Cognitive error in an academic emergency department. Diagnosis 2018;5:135-42.

3. Graber ML, Franklin N, Gordon R. Diagnostic error in internal medicine. Arch Intern Med 2005;165:1493-9.

4. Groopman J. How doctors think. New York: Houghton Mifflin Co, 2007.

5. Sleath J. Dual process theory and bryophyte identification. Field Bryology 2011;105:32-6.

6. Stanovich KE. How to think rationally about world problems. J Intell 2018;6:25.

7. Baron J. Individual mental abilities vs. the world's problems. J Intell 2018;6:23

8. Croskerry P. Our better angels and black boxes. Emerg Med J 2016;33:242-4.

9. Stanovich KE, West RF, Toplak ME. The rationality quotient: toward a test of rational thinking. Cambridge, MA: MIT Press, 2016

10. Croskerry P. Perspectives on diagnostic failure and patient safety. Healthc Q 2012;15:50-6.

11. Graber ML, Kissam S, Payne VL, Meyer AN, Sorensen A, Lenfestey $N$, et al. Cognitive interventions to reduce diagnostic error: a narrative review. BMJ Qual Saf 2012;21:535-57.

12. Croskerry P. Cognitive bias mitigation: becoming better diagnosticians. In: Croskerry P, Cosby K, Graber M, Singh H, editors. Diagnosis: interpreting the shadows. Boca Raton, FL: CRC Taylor and Francis Group, 2017.

13. Lambe KA, O’Reilly G, Kelly BD, Curristan S. Dual-process cognitive interventions to enhance diagnostic reasoning: a systematic review. BMJ Qual Saf 2016;25:808-20.

14. Ludolph R, Schulz PJ. Debiasing health-related judgments and decision making: a systematic review. Med Decis Making 2017;38:3-13. 
15. Higgins S, Hall E, Baumfield V, Moseley D. A meta-analysis of the impact of the implementation of thinking skills approaches on pupils. In: Research evidence in education library. London: EPPICentre, Social Science Research Unit, Institute of Education, University of London, 2005.

16. Norman G. May: a month of myths. Adv Health Sci Educ 2018;23:449-53.

17. Cacioppo JT, Petty RE. The need for cognition. J Pers Soc Psychol 1982;42:116-31.

18. Cazan AM, Indreica SE. Need for cognition and approaches to learning among university students. Procedia Soc Behav Sci 2014;127:134-8.

19. Kahneman D. Thinking fast and slow. New York: Farrar, Straus and Giroux, 2011.
20. Croskerry P. Adaptive expertise in medical decision making. Med Teach 2018;1-6. DOI: 10.1080/0142159X.2018.1484898.

21. Green MJ, Rieck R. Missed it. Ann Intern Med 2013;158:357-61.

22. Dunbar NE, Wilson S, Adame B, Elizondo J, Jensen ML, Miller C, et al. The development of a training game for the mitigation of cognitive bias: the case study of MACBETH. Int J Game-Based Learning 2013;3:7-26.

Pat Croskerry, MD, PhD, Dalhousie University, Critical Thinking, DME, 5849 University Avenue, PO Box 15000, Halifax, Nova Scotia, Canada, Phone: 902-494-4147, Fax: 902-494-2278,

E-mail: croskerry@eastlink.ca 